\title{
Postmortem Time and Storage Temperature Affect the Concentrations of Hypoxanthine, other Purines, Pyrimidines, and Nucleosides in Avian and Porcine Vitreous Humor
}

\author{
EARL E. GARDINER, RUTH C. NEWBERRY, AND JIA-YI KENG \\ Agriculture Canada, Research Station. Agassiz, British Columbia, Canada VOM $1 A O$
}

\begin{abstract}
An HPLC method was used to determine whether postmortem time and storage temperature affect the concentrations of purines, pyrimidines, and nucleosides in avian and porcine vitreous humor. Inosine, hypoxanthine, xanthine, uric acid, uracil, uridine, and thymine were identified in the vitreous humor of chickens (Gallus domesticus). Time from death to sample collection (0-192 h) influenced the concentrations of all seven compounds $(p<$ 0.01 to $<0.0001)$. The storage temperature of chicken carcasses before sampling $\left(6\right.$ or $\left.20^{\circ} \mathrm{C}\right)$ had a significant influence on the concentrations of inosine, hypoxanthine, xanthine, uric acid, uracil, and thymine $(p<0.05$ to $<0.0001$ ). The interaction of postmortem time with temperature was significant for all seven compounds $(p<$ 0.01 ), with the rate of increase in concentration over time being higher at 20 than $6^{\circ} \mathrm{C}$. At $0 \mathrm{~h}$ postmortem, the vitreous humor of pigs (Sus scrofa) contained detectable levels of hypoxanthine, uracil, and uridine. Inosine, hypoxanthine, xanthine, guanosine, guanine, uracil, and uridine were detected after storage of pig eyes for $24 \mathrm{~h}$ at $20^{\circ} \mathrm{C}$. Between 0 and $24 \mathrm{~h}$ postmortem, there was a 22 -fold increase in hypoxanthine concentration and a 13-fold increase in uracil concentration $(p<0.0001)$. It was concluded that postmortem time and temperature are factors that must be taken into consideration when making comparisons of purines, pyrimidines, and nucleosides between groups and in relating postmortem vitreous humor hypoxanthine concentrations to antemortem hypoxia. (Pediatr Res 26:639-642, 1989)
\end{abstract}

\section{Abbreviations}

SIDS, sudden infant death syndrome

PNL, polymorphonuclear neutrophil leucocytes

There is some evidence that the levels of hypoxanthine in plasma, cerebrospinal fluid, and urine are elevated during tissue hypoxia and it has been suggested that a similar elevation occurs in vitreous humor (1). Saugstad and Olaisen (2) investigated the postmortem stability of hypoxanthine concentration in vitreous humor from 86 consecutive legal autopsy cases. They reported that the hypoxanthine level appeared to be independent of sampling time during the first $48 \mathrm{~h}$ postmortem, although significant positive correlations between hypoxanthine concentration and postmortem sampling time were found when times from 0

Received April 24, 1989; accepted August 1, 1989. Correspondence and reprin requests Dr. E. E. Gardiner, Agriculture Canada, Research Station, P.O. Box 1000 Agassiz, British Columbia, Canada, VOM IAO.
90 and $0-192 \mathrm{~h}$ were considered. They concluded that determination of the hypoxanthine concentration in vitreous humor postmortem may be useful in evaluating whether tissue hypoxia preceded circulatory arrest. In a subsequent study (3), elevated levels of hypoxanthine in vitreous humor were reported in victims of SIDS autopsied within $24 \mathrm{~h}$ of death. The SIDS data were compared with those from victims who had died suddenly and violently, and the results interpreted to suggest that death in the SIDS victims was preceded by hypoxia.

Due to certain similarities between SIDS and the sudden death syndrome of broiler chickens (Gallus domesticus) (4), we were interested in using the level of hypoxanthine in vitreous humor to determine if hypoxia is a factor in sudden death syndrome of chickens. In the course of our investigation, we conducted control tests to determine the effects of time and temperature postmortem on hypoxanthine concentrations in the vitreous humor of eyes from healthy chickens. Using an HPLC method of detection, we also obtained data on inosine, xanthine, uric acid, uracil, uridine, and thymine concentrations. In addition, we measured the concentrations of hypoxanthine and some other purines, pyrimidines, and nucleosides in vitreous humor collected from pigs (Sus scrofa) at 0 and $24 \mathrm{~h}$ postmortem. This report presents the results of these analyses.

\section{MATERIALS AND METHODS}

Samples. A total of 120 male cross-bred broiler chickens was reared in accordance with the guidelines of the Canadian Council on Animal Care. At 6 wk of age, the chickens were killed by cervical dislocation. Samples of vitreous humor were collected from 10 of the chickens within 30 min postmortem $(0 \mathrm{~h})$. The remaining 110 carcasses were split into two groups of 55 each. One group was maintained at a room temperature of $20^{\circ} \mathrm{C}$, and the other group at $6^{\circ} \mathrm{C}$. At $4,8,12,24,48,72,96,120,144,168$, and $192 \mathrm{~h}$ postmortem, samples of vitreous humor were obtained from five carcasses in each group. Samples were also obtained from the eyes of ten, 180-d-old pigs slaughtered in a federally inspected abattoir. Before bleeding, the pigs were stunned with electrical current applied behind the ears. One eye was removed from each pig immediately after slaughter. Vitreous humor was collected from five of the eyes within 3 min postmortem $(0 \mathrm{~h})$. The remaining five eyes were held intact at $20^{\circ} \mathrm{C}$ and sampled $24 \mathrm{~h}$ postmortem. Samples were buffered with $0.01 \mathrm{~mol} / \mathrm{L}$ potassium dihydrogen phosphate $(\mathrm{pH} 3.0)$ on collection, filtered, and analyzed immediately.

Chemical analysis. The concentrations of compounds in the vitreous humor were determined by $\operatorname{HPLC}(5,6)$ with the phosphate buffer as eluent. The equipment (Varian Vista 5000 with a model 9090 autosampler combined with a series 601 data system) (Varian Instruments Group, Walnut Creek, CA) was operated at $254 \mathrm{~nm}$ using a $4.6 \times 250 \mathrm{~mm}$ reverse phase C18 
column (Beckman Instruments, Inc., Berkeley, CA). The flow rate was $0.8 \mathrm{~mL} / \mathrm{min}$, with sensitivity $0.01 \AA$ full scale, and the program isocratic. Each compound was identified on the basis of retention time, peak height ratio at 254 versus $280 \mathrm{~nm}$, and coelution with known standards (Sigma Chemical Co., St. Louis, MO). Using this method, the purines hypoxanthine, xanthine, and uric acid, the purine nucleoside inosine, the pyrimidines uracil and thymine, and the pyrimidine nucleoside uridine were identified in vitreous humor from chicken eyes. In the vitreous humor of pigs, the purine guanine and the purine nucleoside guanosine were detected along with hypoxanthine, xanthine, inosine, uracil, and uridine. The retention times in minutes for the compounds were, uracil 5.49 , uric acid 7.75 , guanine 7.99 , hypoxanthine 8.68 , xanthine 10.42 , uridine 11.24 , thymine 13.57, inosine 26.60 , guanosine 29.09 , and the internal standard allopurinol 14.85. In addition to the compounds detected, standards for adenine, cytidine, ortic acid, pseudouridine, and oxypurinol were processed. No peaks in the graphs from the samples were detected for the retention times at which these compounds eluted.

Statistical analysis. All values, except those for inosine and uridine, were transformed to logarithms in order to stabilize the variance and then analyzed by analysis of variance (7). Mean values were back-transformed for presentation. Absolute SEM are presented for inosine and uridine, whereas relative $S E$ are given for the other compounds.

\section{RESULTS}

In chickens, the vitreous humor concentrations of inosine, hypoxanthine, xanthine, uric acid (Table 1), uracil, uridine, and thymine (Table 2 ) were affected by time postmortem (uridine, $p$ $<0.01$; other compounds, $p<0.0001$ ). Hypoxanthine, xanthine, uric acid, uracil, and thymine all showed large increases in concentration with increasing time postmortem. The inosine concentration rose to a peak and then dropped, whereas uridine levels showed no obvious pattern over time.

The storage temperature of the chicken carcasses prior to sampling influenced the concentrations of inosine, hypoxanthine, xanthine, uric acid, uracil, and thymine (uric acid, $p<$ 0.05 ; inosine, $p<0.01$; other compounds, $p<0.0001$ ). In most cases, concentrations were higher in vitreous humor from car-

Table 1. Mean concentrations ( $\mu$ mol/L) of inosine, hypoxanthine, xanthine, and uric acid in the vitreous humor of 6-wk-old chickens: effects of postmortem sampling time and carcass storage temperature

\begin{tabular}{|c|c|c|c|c|c|c|c|c|}
\hline \multirow{2}{*}{$\begin{array}{l}\text { Postmortem } \\
\text { Time* } \\
\text { (h) }\end{array}$} & \multicolumn{2}{|c|}{ Inosine } & \multicolumn{2}{|c|}{ Hypoxanthine } & \multicolumn{2}{|c|}{ Xanthine } & \multicolumn{2}{|c|}{ Uric ació } \\
\hline & $6^{\circ} \mathrm{C}$ & $20^{\circ} \mathrm{C}$ & $6^{\circ} \mathrm{C}$ & $20^{\circ} \mathrm{C}$ & $6^{\circ} \mathrm{C}$ & $20^{\circ} \mathrm{C}$ & $6^{\circ} \mathrm{C}$ & $20^{\circ} \mathrm{C}$ \\
\hline 0 & & 10 & & 16 & & 3 & & 39 \\
\hline 4 & 18 & 24 & 41 & 63 & 10 & 12 & 29 & 28 \\
\hline 8 & 30 & 54 & 55 & 124 & 10 & 23 & 30 & 45 \\
\hline 12 & 39 & 35 & 70 & 153 & 14 & 29 & 35 & 33 \\
\hline 24 & 34 & 49 & 109 & 263 & 22 & 48 & 47 & 59 \\
\hline 48 & 41 & 30 & 227 & 498 & 49 & 131 & 76 & 143 \\
\hline 72 & 46 & 26 & 361 & 688 & 89 & 259 & 142 & 272 \\
\hline 96 & 50 & 18 & 497 & 875 & 128 & 404 & 184 & 328 \\
\hline 120 & 39 & 15 & 678 & 937 & 212 & 509 & 352 & 369 \\
\hline 144 & 31 & 19 & 670 & 966 & 232 & 657 & 399 & 360 \\
\hline 168 & 28 & 19 & 872 & 1058 & 325 & 927 & 457 & 423 \\
\hline 192 & 20 & 26 & 801 & 1014 & 345 & 932 & 470 & 229 \\
\hline SEM + & \multicolumn{2}{|c|}{43} & \multicolumn{2}{|c|}{0.06} & \multicolumn{2}{|c|}{0.14} & \multicolumn{2}{|c|}{0.11} \\
\hline
\end{tabular}

* $0 \mathrm{~h}, n=10$ chickens; $4-192 \mathrm{~h}, n=5$ chickens.

$\dagger$ Inosine, absolute means, and SEM; other compounds, back-transformed means, and relative SEM (to obtain SE for a given mean, multiply mean by relative SEM).
Table 2. Mean concentration ( $\mu \mathrm{mol} / \mathrm{L}$ ) of uracil, uridine, and thymine in vitreous humor of 6-wk-old chickens: effects of postmortem sampling time and carcass storage temperature

\begin{tabular}{|c|c|c|c|c|c|c|}
\hline \multirow{2}{*}{$\begin{array}{l}\text { Postmortem } \\
\text { Time** } \\
\text { (h) }\end{array}$} & \multicolumn{2}{|c|}{ Uracil } & \multicolumn{2}{|c|}{ Uridine } & \multicolumn{2}{|c|}{ Thymine } \\
\hline & $6^{\circ} \mathrm{C}$ & $20^{\circ} \mathrm{C}$ & $6^{\circ} \mathrm{C}$ & $20^{\circ} \mathrm{C}$ & $6^{\circ} \mathrm{C}$ & $20^{\circ} \mathrm{C}$ \\
\hline 0 & \multicolumn{3}{|c|}{0.8} & 16 & & $\mathrm{ND}_{\ddagger}^{\ddagger}$ \\
\hline 4 & 18 & 22 & 26 & 26 & ND & ND \\
\hline 8 & 24 & 52 & 25 & 34 & 0.2 & ND \\
\hline 12 & 35 & 55 & 28 & 28 & 0.7 & ND \\
\hline 24 & 57 & 109 & 31 & 29 & ND & ND \\
\hline 48 & 90 & 208 & 23 & 19 & ND & ND \\
\hline 72 & 151 & 335 & 31 & 20 & ND & 4.8 \\
\hline 96 & 186 & 530 & 26 & 27 & ND & 34 \\
\hline 120 & 279 & 709 & 24 & 21 & 1.6 & 51 \\
\hline 144 & 291 & 863 & 24 & 19 & 11 & 131 \\
\hline 168 & 387 & 1166 & 38 & 9 & 15 & 196 \\
\hline 192 & 394 & 1178 & 22 & 34 & 19 & 255 \\
\hline SEM $\ddagger$ & \multicolumn{2}{|c|}{0.13} & \multicolumn{2}{|c|}{3.7} & \multicolumn{2}{|c|}{0.24} \\
\hline
\end{tabular}

$* 0 \mathrm{~h}, n=10$ chickens; $4-192 \mathrm{~h}, n=5$ chickens.

$\dagger$ Not detected (i.e. concentration $<0.25 \mu \mathrm{mol} / \mathrm{L}$ ).

\$ Uridine, absolute means and SEM; other compounds, back-transformed means and relative SEM (to obtain SE for a given mean, multiply mean by relative SEM).

casses stored at 20 than at $6^{\circ} \mathrm{C}$ (Tables 1 and 2). Storage temperature did not influence uridine levels $(<0.12)$. The postmortem time $\times$ temperature interaction was significant for all compounds ( $p<0.01$, Tables 1 and 2 ). The rate of increase in the concentrations of the compounds over time was higher at 20 than $6^{\circ} \mathrm{C}$. The ratio of xanthine/hypoxanthine was calculated for all individual chickens and ranged from 0.14 to 1.09 .

In porcine vitreous humor (Table 3 ), the only compounds detected at $0 \mathrm{~h}$ postmortem were hypoxanthine, uracil, and uridine. At $24 \mathrm{~h}$ postmortem, inosine, xanthine, guanosine, and guanine were also detected. Between 0 and $24 \mathrm{~h}$ postmortem, the hypoxanthine concentration increased 22-fold $(p<0.0001$ ). The level of uracil also increased $(p<0.0001)$, whereas postmortem time had no effect on uridine concentration $(p<0.54)$.

\section{DISCUSSION}

Although the concentration of the nucleoside inosine, the precursor of the purines hypoxanthine, xanthine, and uric acid (8), was affected by postmortem time and temperature, the changes in concentration were small by comparison with those of the three purines. This suggests that the conversion of inosine to hypoxanthine was inhibited to only a slight extent by postmortem conditions in the eye of the chicken. After the initial increase in inosine (slower at 6 than $20^{\circ} \mathrm{C}$ ), the concentration decreased and became relatively constant after $72 \mathrm{~h}$ for the $20^{\circ} \mathrm{C}$ samples, suggesting a decreased supply of inosine from adenosine or a more rapid conversion to hypoxanthine or both. We could find no other published reports on inosine levels in vitreous humor for comparison with our data. O'Connor et al. (9) reported inosine levels of $0.22 \mu \mathrm{mol} / \mathrm{L}$ in umbilical arterial plasma of well-oxygenated fetuses and $0.27 \mu \mathrm{mol} / \mathrm{L}$ in birth asphyxiated infants.

The increase in concentrations of hypoxanthine, xanthine, and uric acid with increasing time postmortem indicates that the enzymatically catalyzed reactions involved in their production were occurring for a considerable time postmortem. At different times (depending on the temperature), a maximum concentration of each purine was reached, suggesting that certain reactions had stopped at these times. These results do not support the statement (3), incorrectly attributed to Bill (11), that "vitreous humor is metabolically stable due to the lack of enzymes." 